Eurasian Journal of Physics and Functional Materials

$2021,5(4), 169-180$

\title{
A comparative study of promising filter materials for neutron imaging facilities
}

\author{
K.M. Nazarov*,1,2,3, S.E. Kichanov ${ }^{1}$, E.V. Lukin ${ }^{1}$, \\ I.Yu. Zel ${ }^{1}$, D.P. Kozlenko ${ }^{1}$, T.K. Zholdybayev ${ }^{3,4}$, \\ B. Muhametuly ${ }^{1,3,4}$, M. Kenessarin ${ }^{3,4}$, A.V. Rutkauskas ${ }^{1}$, \\ A. Yskakov ${ }^{1,2,3}$, M.O. Belova ${ }^{1}$ \\ ${ }^{1}$ Joint Institute for Nuclear Research, Dubna, Russia \\ ${ }^{2}$ L.N. Gumilyov Eurasian National University, Nur-Sultan, Kazakhstan \\ ${ }^{3}$ The Institute of Nuclear Physics Ministry of Energy of the Republic of Kazakhstan, Almaty, Kazakhstan \\ ${ }^{4}$ Al-Farabi Kazakh National University, Almaty, Kazakhstan \\ E-mail: knazarov@jinr.ru
}

DOI: $10.32523 /$ ejpfm.2021050401

Received: 18.11.2021 - after revision

The effect of sapphire and bismuth single-crystal filters and their combinations on the quality of neutron radiographic images and neutron tomography data has been studied. The parameters of the contrast of the neutron image were analyzed depending on the monocrystalline filters. Neutron transmission spectra were obtained for sapphire and bismuth single crystals. Additionally, the effect of filters on the overall intensity of the thermal neutron beam and the background of gamma-rays was investigated. Based on the obtained data, we assume that a single-crystal sapphire filter can be most effectively used for radiographic and tomographic installations using thermal neutrons.

Keywords: neutron radiography, tomography, single-crystal filters, sapphire, bismuth, image quality.

\section{Introduction}

In recent decades, there has been a growing interest in the development, design, and parameters optimization of neutron radiography and tomography facilities 
in the world's neutron centers. Firstly, neutron imaging is a powerful nondestructive testing technique [1,2]. The difference in the nature of the interaction of neutrons with a matter in comparison with X-rays gives additional advantages to neutron methods, including sensitivity to light elements and isotopes, the difference in contrast between neighboring elements, and deep penetration into bulk objects. All these features make neutron radiography and tomography highly demanded tools with a growing range of applications in nuclear industry [3], building materials [4, 5], lithium-ion batteries [6], geophysics [7], paleontology and archaeology [8], and other various fields. On the other hand, the relative budget and simplicity of implementation of the neutron radiography method on various types of neutron sources lead to the development of neutron imaging facilities as a project of the first stage realization at the different neutron centers $[9,10]$. In this context, the steady trend of the development of neutron imaging instrumentation on reconstructed or updated nuclear reactors should be noted. As an example, the new well-advanced neutron imaging facilities on the IR-8 reactor (Moscow, Russia) [11], BRR reactor (Budapest, Hungary) [12], WWR-K research reactor (Almaty, Republic of Kazakhstan) [13, 14], LVR-15 reactor (Rez, Czech republic) [15], NCNR reactor (Gaithersburg, USA) [16] and IBR-2 high-flux pulsed reactor (Dubna, Russia) $[17,18]$ are being put into operation. One of the features of these neutron facilities is using a collimator system that forms a neutron beam for radiography and tomography experiments. In comparison with modern advanced neutron imaging facilities with curved neutron guides [19-22], the direct-of-sight collimator system provides a neutron beam with a high admixture of fast neutrons as well as gamma rays. This causes rapid degradation of the CCD-chip of camera and electronics of the detector system and irradiation of other facility components. The use of single-crystal neutron filters is the simplest and relatively cheap solution to reduce the concomitant unwanted radiations of the neutron beam. Several computational and experimental works are devoted to the problem of optimization and choosing of single-crystal filters [23, 24], like silicon [25], quartz [26], sapphire [27-29], bismuth [30], and lead [31]. However, there is a lack of experimental data on the performance or efficiency of neutron filters concerning the neutron imaging data. In our work, we present the results of measurements of an effect of sapphire and bismuth single-crystal filters on the quality of neutron imaging data.

\section{Experimental part}

\section{The description of the single-crystal neutron filters}

Several single-crystal sapphire and bismuth samples were selected for measurements, the real photo of which is shown in Figure 1. The sizes of cylindrical sapphire and parallelepiped bismuth filters are listed in Table 1. In all experimental measurements, the crystallographic axis (111) of bismuth and sapphire single crystals was parallel to the direction of the incident neutron beam. 
Table 1.

Size of the neutron filters

\begin{tabular}{c|ccc}
\hline Filter material & Sapphire & Bismuth & Bismuth \\
\hline Size & $50 \times 100 \mathrm{~mm}$ & $60 \times 60 \times 20 \mathrm{~mm}$ & $60 \times 68 \times 30 \mathrm{~mm}$ \\
\hline
\end{tabular}

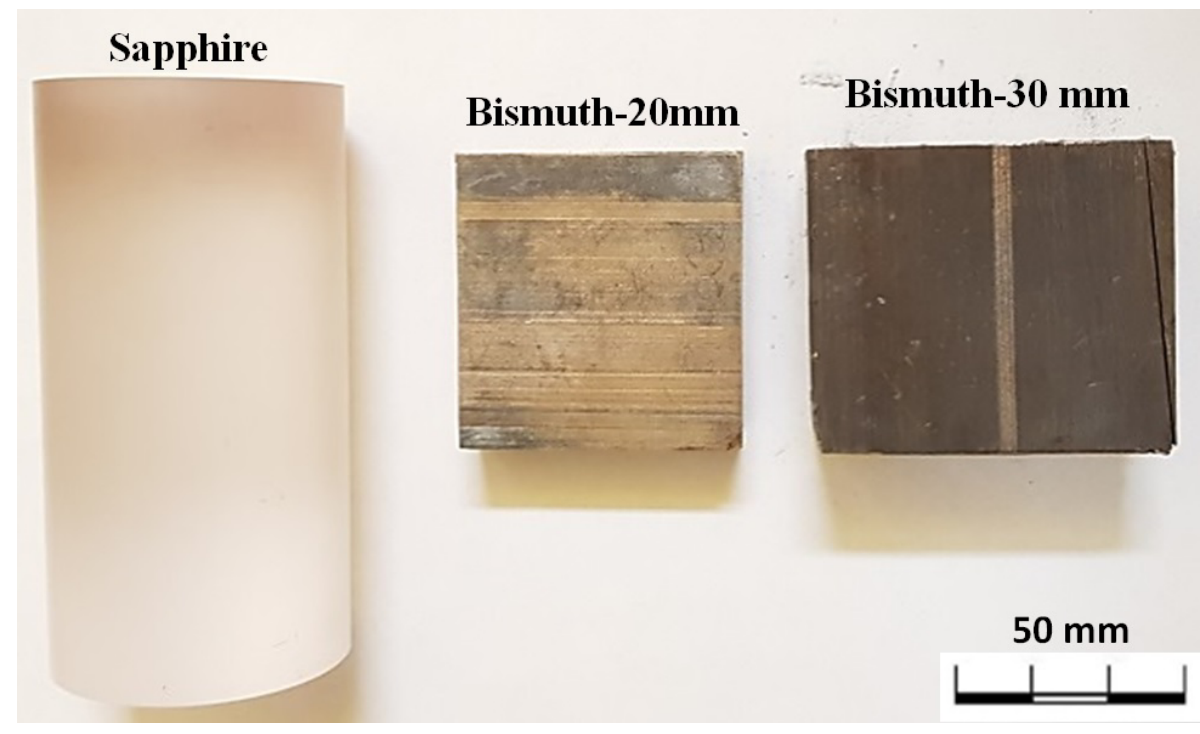

Figure 1. The photography of the single-crystal filters used in the neutron imaging experiments.

For neutron imaging experiments, we used both separate fragments of each single-crystal filter and their combinations, which description and labeling, used below, is given in the Table 2. With the aim of simplicity of implementation or modification of the neutron filter block and their easy replacement, all experiments were carried out at room temperature.

Table 2.

The labeling of the filter configuration used in the neutron measurements.

\begin{tabular}{l|l}
\hline Label & Filter configuration \\
\hline 1 & without a filter \\
\hline 2 & Bismuth, thickness $=20 \mathrm{~mm}$ \\
\hline 3 & Bismuth, thickness $=30 \mathrm{~mm}$ \\
\hline 4 & Bismuth, thickness $=50 \mathrm{~mm}$ \\
\hline 6 & Sapphire, thickness $=100 \mathrm{~mm}$ \\
\hline 7 & Sapphire + Bismuth, thickness $=20 \mathrm{~mm}$ \\
\hline
\end{tabular}

\section{Neutron radiography and tomography measurements}

The experiments were performed at the neutron radiography and tomography facility NRT placed on beamline 14 of the IBR-2 high-flux pulsed reactor $[17,18]$. The incident thermal neutron beam has a spectral distribution maximum of 1.8 Aand covers wavelength range from 0.2 to $8 \AA$. The neutron flux at the sample position is $\approx 5.5(2) \times 10^{6} \mathrm{n} / \mathrm{cm}^{2} / \mathrm{s}$. The distance from the moderator (light water, $300 \mathrm{~K}$ ) to the detector system of the setup is $23.5 \mathrm{~m}$, and the collimation parameter was the same in all experiments $L / D=380$. The main components 
of the experimental setup NRT are shown in Figure 2. In our measurements, we used the CCD-camera based detector system with the chip HAMAMATSU S12101 and LiF/ZnS:Ag scintillation screen with a thickness of $100 \mu \mathrm{m}$. The average neutron image was formed by the summing of five separate images using a Z-projection median filter [32]. The imaging data were corrected by the dark current image and normalized to the image of the incident neutron beam using the ImageJ software [33]. The test neutron tomography experiments were performed using the system by rotating the sample through $180^{\circ}$ and with a step of $0.5^{\circ} .360$ neutron radiographic images for the different angular position of the test sample relative to the neutron beam direction was used for the tomography reconstruction procedure, which was performed by the SYRMEP Tomo Project software [34].

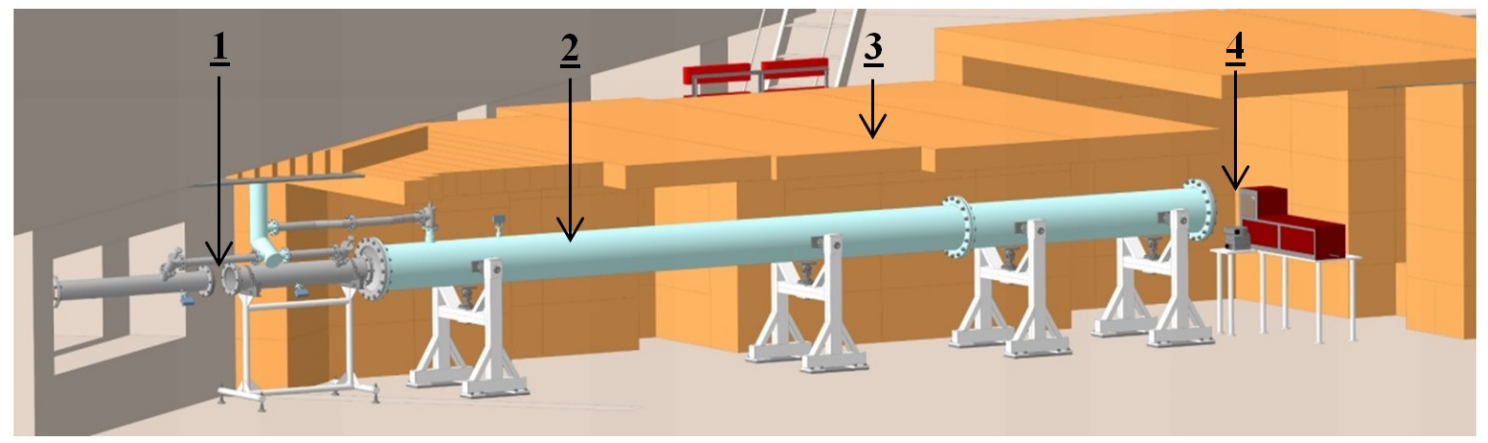

Figure 2.Layout of neutron radiography and tomography instrument NRT of IBR-2 pulsed reactor: 1 - position for neutron filter block, 2 - evacuated flight tube, 3 - biological shielding, 4 - sample rotating stage and detector system position.

The quality of neutron radiographic images was characterized through several standard parameters [35]. These are the spatial resolution, signal-noise ratio (SNR) and the contrast of radiographic images [36]. In neutron radiography, a plane-parallel beam from a source is used, which is created by a slit of a finite size, the spatial resolution of the image mainly depends on the collimation of the beam or the L/D ratio. The use of filters does not particularly affect the collimation of the beam, and therefore the geometric blur. The SNR of a neutron image mainly depends on the number of neutrons or statistics. In our experiments, the same exposure time was set to obtain a neutron image. Therefore, this means that different intensities of neutrons fall on the detector. This makes it difficult to compare the efficiency of neutron filters. Due to the above reasons, in this work, changes in SNR parameters and spatial resolution were not considered. We evaluated the effect of additional filters on the contrast parameter, which significantly affects the quality of neutron radiography and tomography images. Image contrast is the appearance of a difference in a grayscale of the resulting image between closely spaced materials with different attenuation coefficients. The contrast parameter was defined as follows:

$$
\text { Contrast }=\frac{I_{a}-I_{b}}{\left.I_{f}+I_{b}\right)}
$$

where $I_{a}$ and $I_{b}$ are the intensities of the image pixels corresponded to different materials or densities of the tested objects. The neutron attenuation coefficient $\mu$ 
for studied samples is determined by the Beer-Lambert formula:

$$
\mu=-\ln \left(\frac{I_{t}}{I_{0}}\right)
$$

where $I_{O}$ is the initial intensity the beam, $I_{t}$ is the transmitted intensity.

\section{The measurements of neutron transmission spectra}

The neutron transmission spectra corresponding to different single-crystal filters were measured using the DN-6 diffractometer at the IBR-2 pulsed reactor [37]. The neutron scattering patterns of the vanadium sample with a volume of 75 $\mathrm{mm}^{3}$ were measured by the time-of-flight mode at fixed scattering angle of $2 \theta=$ $90^{\circ}$ with an exposure time of $15 \mathrm{~min}$. Single crystals of filters were placed in front of the vanadium sample at a distance of $1.2 \mathrm{~m}$. The obtained neutron spectra were normalized to the initial vanadium spectrum.

\section{Results and discussions}

\section{Neutron transmission spectra}

The obtained neutron transmission spectra of different single-crystal filters are shown in Figure 3. For the single-crystal sapphire filter, a sharp increase in the neutron transmission curve from $53 \%$ to $80 \%$ is observed in the range of the neutron wavelengths of $0.8-2 \AA$. For the cold neutrons region $3.2 \AA-8 \AA$, a monotonic decrease in the sapphire transmission curve from $78 \%$ to $58 \%$ is observed. The neutron transmission spectra of the single-crystal bismuth filters of different thickness of $20 \mathrm{~mm}$ and $50 \mathrm{~mm}$ are shown in Figure 3. A rather smooth neutron transmission spectrum is observed, and the transmission level is $93-98 \%$ for the neutron wavelength range $4-8 \AA$. With an increase of bismuth thickness to $50 \mathrm{~mm}$, some decrease in the intensity of transmitted neutrons occurs. In the range of short wavelengths neutrons from 1 to $4 \AA$, several dips on the transmission curve appear due to losses caused by diffraction scattering with the mosaicity of a bismuth crystal with a thickness of $30 \mathrm{~mm}$.

The next step was to study the effect of filters on the attenuation of the thermal neutron beam intensity and gamma-ray background was investigated. The intensity of thermal neutrons was estimated by the intensity value of the image pixels of an empty open beam with various filter configurations (see Table 1) were obtained by a detector based on a scintillation screen and a CCD camera. A comparative diagram of the decrease in the total intensity of thermal neutrons and the background of gamma rays for various filter configurations is shown in Figure 4 .

As can be seen from the diagram, the use of filters reduces the overall intensity of the neutron beam, for example, up to 2.5 times for filter configuration 4 with a $50 \mathrm{~mm}$ bismuth filter and up to 3 times for configuration 7 . It is interesting to note that the gamma background level of the open beam also decreases with 


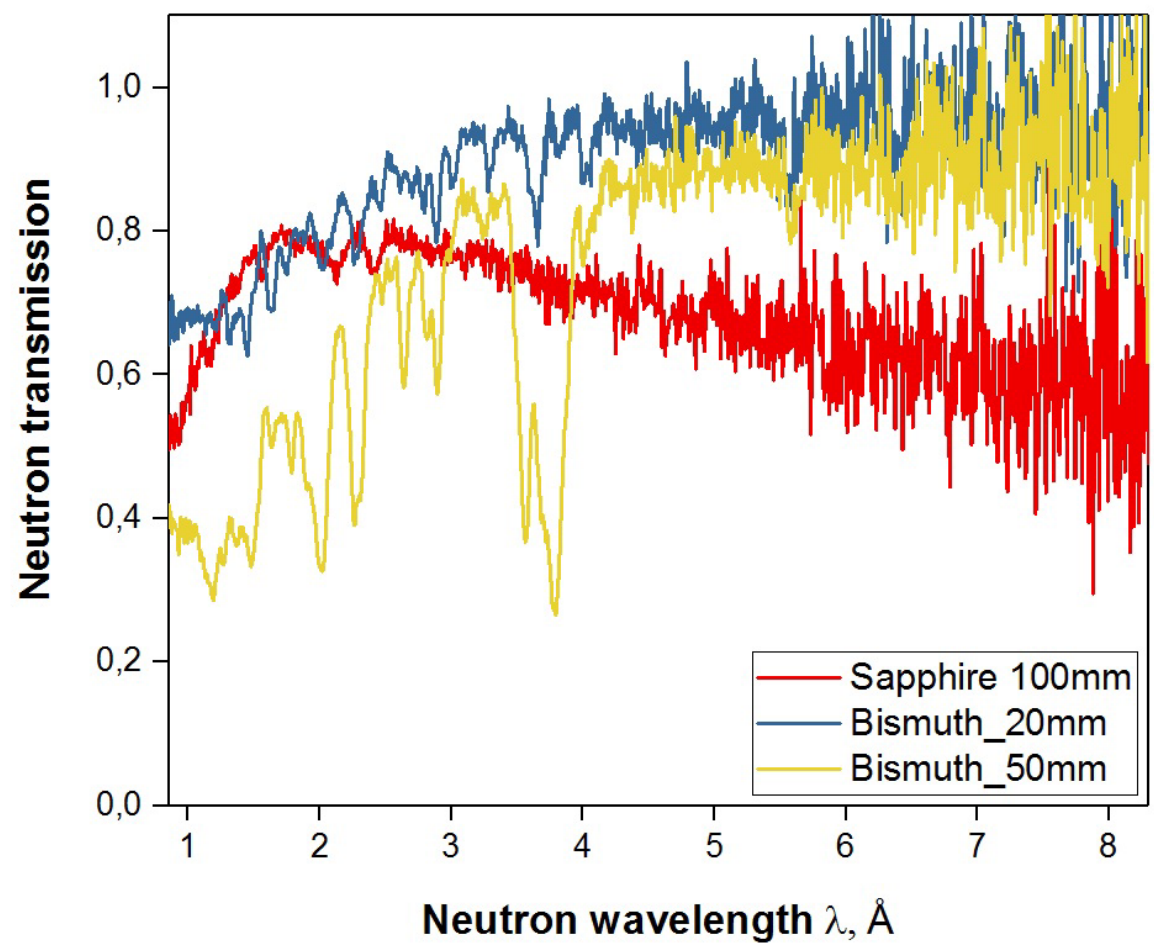

Figure 3. Neutron transmission spectra from different single-crystal filters: (red) sapphire with a thickness of $100 \mathrm{~mm}$, (blue) bismuth with a thickness of $20 \mathrm{~mm}$, (yellow) bismuth with a thickness of $50 \mathrm{~mm}$.

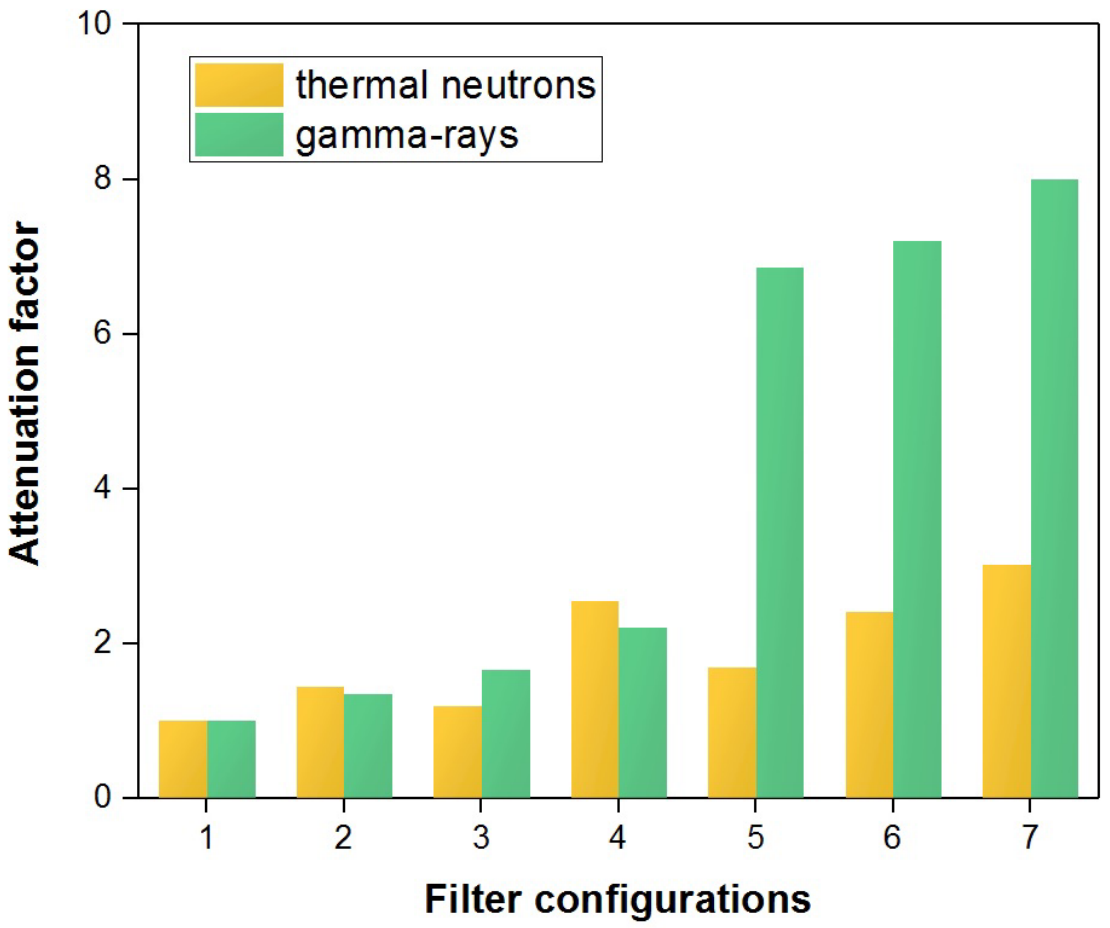

Figure 4. Comparative diagram of changes in the attenuation of the intensity of thermal neutrons and gamma-ray background depending on the different configurations of filters. The relative values are given normalized to the data for configuration 1 without a filter.

adding filters. Despite the fact that the bismuth filter is the main filter for gammaray suppression, the sapphire filter contributes more to the improvement of the background state near the detector system and the experimental facility as a 
whole. This indicates that initially the neutron flux from the reactor moderator is not fully thermalized and fast neutrons predominate in the beam in large quantities. Then fast neutrons hitting the protective elements and the collimator system of the facility enter into reactions and, as a result, create an undesirable background from gamma rays. When using a sapphire filter (configuration 5), a 6.8-fold decrease in gamma rays is observed, while the intensity of thermal neutrons decreases by only $40 \%$ or 1.6 times. The combination of bismuth filters with a sapphire filter (configuration 6 and 7) improves the radiation state insignificantly and gives a decrease in the gamma background by 7.2 and 8.1 times, respectively.

\section{Contrast study at the neutron radiography experiments}

In this part of the work, the change in contrast parameters in neutron images obtained using various filters was investigated. We had selected several model test objects from different materials with different neutron attenuation coefficients to compare the observed effect for high-, moderate- and low-neutron-attenuated materials. There are four metal cylinders with a diameter of $13.5 \mathrm{~mm}$ (Figure 5a). The materials for cylinders were lead, aluminum, copper and iron. To reduce the effect of scattered neutrons, in all measurements the sample was located at a distance of $4.8 \mathrm{~cm}$ from the detector. The example of the neutron radiography image of tested metal objects is shown in Figure 5b. The yellow and red rectangles label the selection areas used to calculate the contrast parameter in the experimental tests. The signal intensity was determined for a rectangular area of $20^{*} 4 \mathrm{~mm}^{2}$ in the central part of cylindrical samples (colored yellow in Figure 5), and the standard deviation was determined for an area of $20 \cdot 4 \mathrm{~mm}^{2}$ near these samples (colored red in Figure 5).
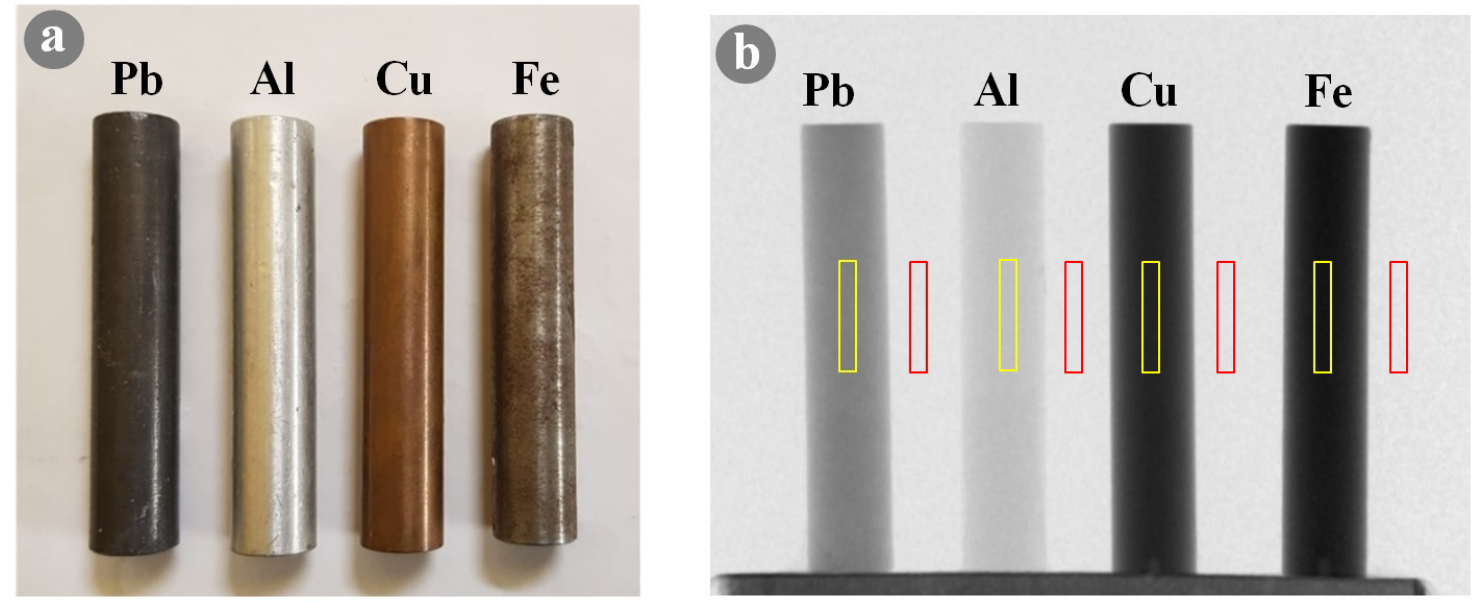

Figure 5. Photo (a) and neutron radiography image (b) of the cylindrical samples of lead, aluminum, copper and iron. The yellow and red rectangles (with dimension $20 \cdot 4 \mathrm{~mm}^{2}$ ) label the selection areas used to calculate the contrast parameters in the experimental tests.

An average value of attenuation coefficients and contrast parameters for various metallic materials depending on filter configuration is shown in Table 3. For tested materials with a low attenuation coefficient, like aluminum, inserting 
filters on the beam improves the attenuation coefficients and the contrast parameter, but all changes are at the statistical error level. For a lead sample, a high attenuation coefficient appears only in configuration 1, when there is no filter. In other configurations, the values of the attenuation coefficients and the contrast parameters decrease, due to the fact that the filtered beam reduces a large fraction of gamma rays. At the same time, for iron and copper samples, an improvement in these parameters is observed for all filters used. This is due to an additional attenuation of the intensity of fast neutrons and gamma-rays of the beam by filtering materials. The maximum attenuation and contrast coefficients for lead and copper samples correspond to configuration 6, which used a combination of sapphire and bismuth filters. As an illustration, Figure 6 shows a comparative graph of the change in the value of the neutron attenuation coefficients for the iron sample at various configurations.

Table 3.

The average values of the $\mu$ attenuation coefficients and C contrast parameters of radiographic images for metal samples depending on the different configurations of filterss.

\begin{tabular}{|l|l|c|c|c|c|c|c|c|}
\hline Sample & \multicolumn{2}{|c|}{$\mathrm{Pb}$} & \multicolumn{2}{c|}{$\mathrm{Al}$} & \multicolumn{2}{c|}{$\mathrm{Cu}$} & \multicolumn{2}{c|}{$\mathrm{Fe}$} \\
\hline Label & $\mu$, & $\mathrm{C}$ & $\mu$ & $\mathrm{C}$ & $\mu$ & $\mathrm{C}$ & $\mu$ & $\mathrm{C}$ \\
\hline 1 & 0.268 & 0.193 & 0.072 & 0.0606 & 0.702 & 0.456 & 0.801 & 0.506 \\
\hline 2 & 0.259 & 0.187 & 0.073 & 0.0613 & 0.723 & 0.466 & 0.826 & 0.516 \\
\hline 3 & 0.254 & 0.183 & 0.074 & 0.0608 & 0.734 & 0.472 & 0.830 & 0.519 \\
\hline 4 & 0.252 & 0.178 & 0.079 & 0.0602 & 0.752 & 0.479 & 0.852 & 0.526 \\
\hline 5 & 0.259 & 0.186 & 0.077 & 0.0610 & 0.759 & 0.484 & 0.878 & 0.543 \\
\hline 6 & 0.258 & 0.182 & 0.080 & 0.0613 & 0.787 & 0.495 & 0.906 & 0.554 \\
\hline 7 & 0.248 & 0.177 & 0.078 & 0.0612 & 0.786 & 0.495 & 0.903 & 0.549 \\
\hline
\end{tabular}

\section{Contrast study at the neutron radiography experiments}

The effects from the filters and their configurations on neutron tomography data were studied using a test object (Figure 7a) similar to the corresponding one from the PSI NIQA samples kit [38]. It is an aluminum cylinder with six inserts of different materials. There are copper, lead, aluminum, nickelsilver alloys, iron and empty hole. This test sample is designed to test the ability to qualitatively determine the contrast parameters and neutron attenuation coefficients for various materials using tomography processes. The neutron tomography contrast parameter was obtained by averaging the voxel values over the entire volume of the cylindrical inserts (Figure $7 \mathrm{~b}$ ). For the contrast parameters calculation using Eq. (1), several regions of interest (ROI) of the tomography slice were chosen (Figure 7c). The obtained contrast parameters were presented in comparison to corresponding parameter of the aluminum material. To measure the value of the signal $I_{a}$, a circular region of interest was selected, which includes most of it while avoiding the edges. The attenuation coefficient of the aluminum cylinder was chosen as the values of the adjacent region $\left(I_{b}\right)$, which was measured as the average value of the intensity of the voxels in four square regions of interest located near the circular region $I_{a}$. 


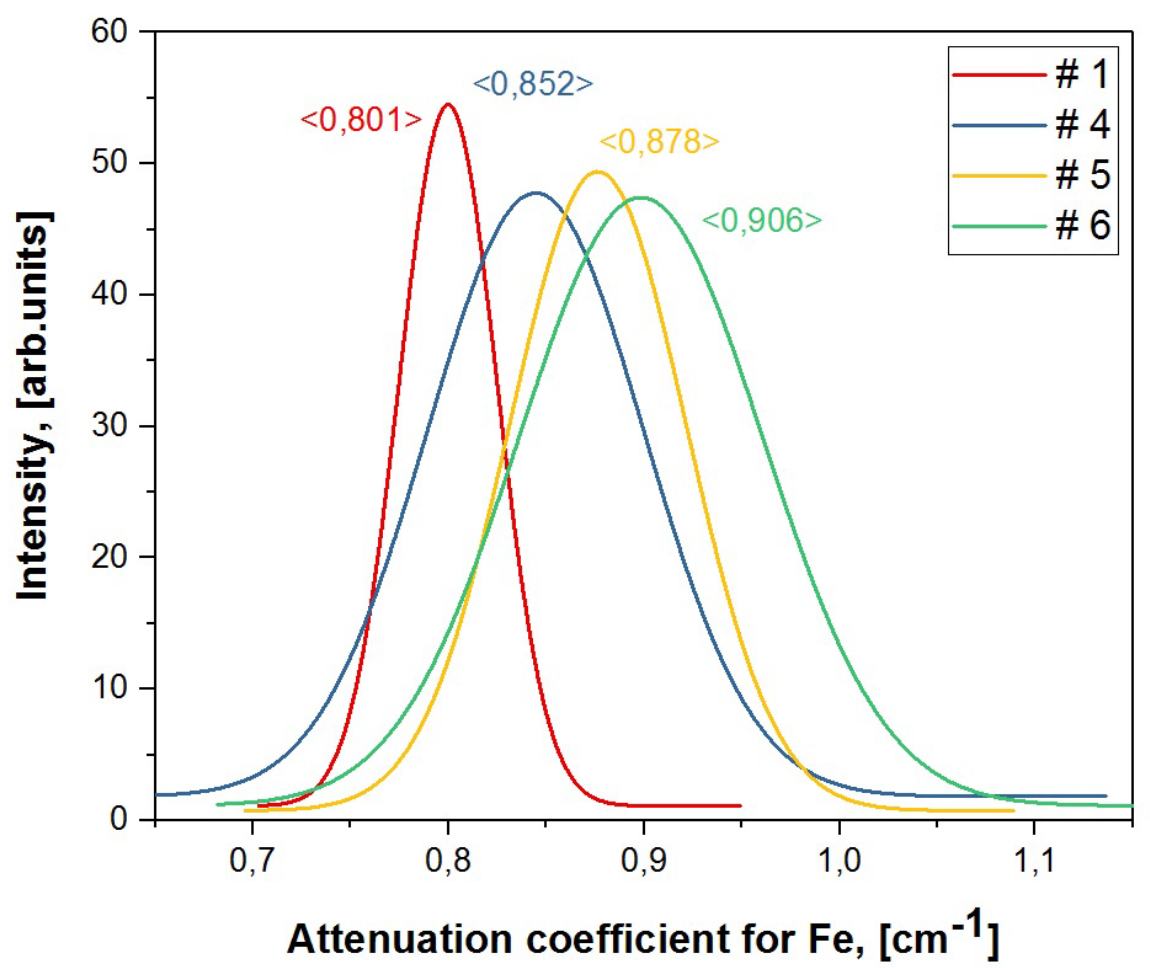

Figure 6. Comparative graph of the change in the value of the neutron attenuation coefficients for the iron sample at various configurations.
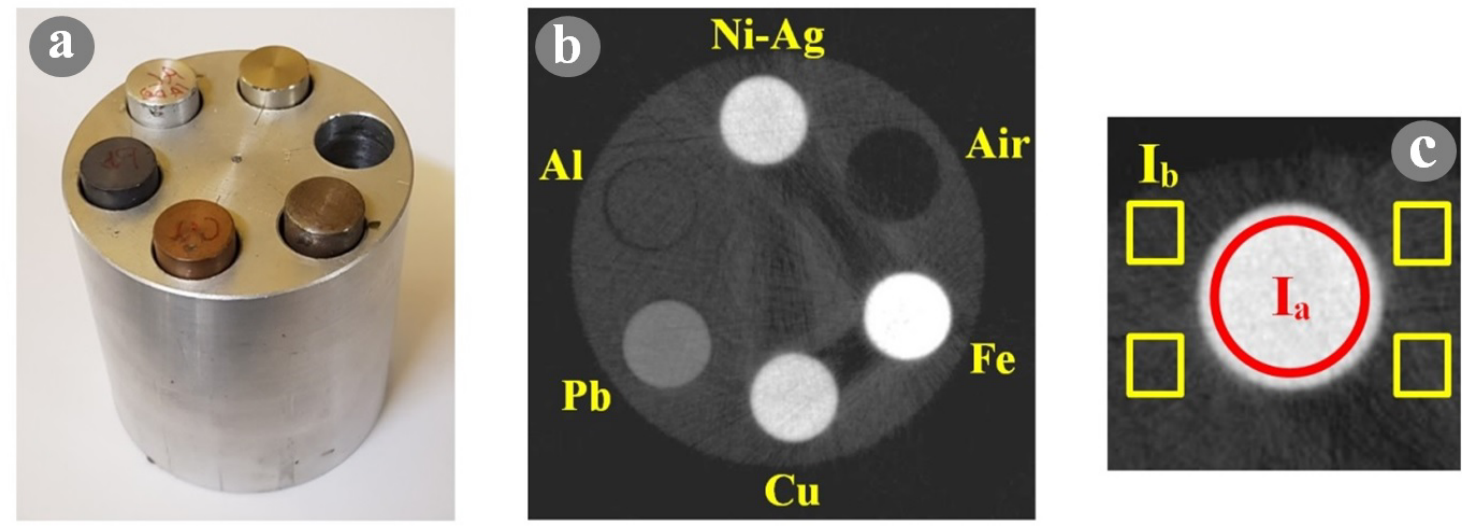

Figure 7. Photo (a) and the slice (b) of the 3D model reconstructed from the tomography data of a model cylindrical sample. The example of circular ROIs (c) corresponding to $I_{a}$ and $I_{b}$ for the contrast parameters calculations using Eq.1. The corresponding parameter Ia is the average number of the pixels of the circular region A (red label), the intensity $\mathrm{Ib}$ corresponds the average number of the pixels of restricted area labeled by yellow circle.

Attenuation coefficient and contrast values calculated from neutron tomography data for metal rods using various filter configurations are shown in Table 4. As can be seen from the table, for metals with a high attenuation coefficient (copper, iron and nickel-silver alloy), an increase in the contrast parameter is observed with an increase in the attenuation coefficient when filters are added. At the same time, the opposite effect is observed for a lead sample, the values of both parameters decrease with the addition of filters. High values of the observed parameters are achieved only in configuration 1, when there is no filter in the beam. The contrast parameter between aluminium and voids (air) is maximum in configuration 6 , but the changes in the attenuation coefficient are practically 
insignificant. Compared to the others, the configuration of filter 6 gives a noticeable increase in the parameter for all materials except lead. As can be seen from the data, the combination of sapphire and bismuth filter provides an increase in the neutron attenuation coefficient on average by $14-22 \%$, while the same filter increases the contrast between aluminium and metal inserts by about 3-6\%.

Table 4 .

The average values of the contrast parameters and neutron attenuation coefficients calculated from tomographic data of the test sample with different metal rods for different configurations of filters.

\begin{tabular}{|l|l|c|c|c|c|c|c|c|c|c|}
\hline Sample & \multicolumn{2}{|c|}{$\mathrm{Pb}$} & \multicolumn{2}{c|}{$\mathrm{Ni}-\mathrm{Ag}$} & \multicolumn{2}{c|}{$\mathrm{Cu}$} & \multicolumn{2}{c|}{$\mathrm{Fe}$} & \multicolumn{2}{c|}{ Air } \\
\hline Label & $\mu$ & $\mathrm{C}$ & $\mu$ & $\mathrm{C}$ & $\mu$ & $\mathrm{C}$ & $\mu$ & $\mathrm{C}$ & $\mu$ & $\mathrm{C}$ \\
\hline 1 & 0.270 & 0.505 & 0.673 & 0.766 & 0.661 & 0.763 & 0.808 & 0.801 & 0.016 & 0.688 \\
\hline 4 & 0.254 & 0.480 & 0.733 & 0.783 & 0.724 & 0.780 & 0.864 & 0.813 & 0.018 & 0.661 \\
\hline 5 & 0.262 & 0.495 & 0.736 & 0.785 & 0.720 & 0.780 & 0.880 & 0.817 & 0.016 & 0.687 \\
\hline 6 & 0.262 & 0.492 & 0.827 & 0.805 & 0.790 & 0.797 & 0.8936 & 0.832 & 0.014 & 0.713 \\
\hline
\end{tabular}

The choice of filter material for thermal neutron beams depends on many factors: the distance between source and detector, the location of the beams, and the type of unwanted radiation (gamma-rays or fast neutrons). For fast neutron suppression, a sapphire filter may be the preferred option, a bismuth filter for the gamma-ray component, and a combination of bismuth and sapphire filters for both. In most cases, a sapphire filter (10-15 cm thick) can be effectively used for most neutron radiography and tomography stations as a cheaper option than a single crystal bismuth filter. Based on our data, it can be argued that single-crystal sapphire is more preferable as a neutron filter for use on a thermal neutron beam where short wavelength neutrons prevail. In addition, the effect of attenuation of gamma radiation when using a sapphire filter is observed, which makes it possible to reduce the background of secondary gamma-rays appearing when fast neutrons interact with the radiation-protective elements of the experimental setup. Furthermore, as shown by our measurements, after the main sapphire filter, a thin filter for gamma-rays such as bismuth can be added which improves the quality of radiographic and tomographic images. The additional $20 \mathrm{~mm}$ thickness of bismuth is sufficient to suppress the low energy gamma-rays that appear after fast neutron scattering with sapphire. Consequently, the combination of sapphire with an additional thin bismuth filter can improve the contrast of the image, as well as increase the neutron attenuation coefficient for good identification of materials in tomographic measurements.

\section{Conclusion}

In our work, we investigated the effects of single-crystal sapphire and bismuth filters, as well as their configurations, on the quality of neutron imaging data, including radiography and tomography. The neutron transmission spectra of these filters were obtained. Their influence on the quality parameters of neutronradiographic images and tomographic data has been studied in detail. In most 
cases, a sapphire filter can be effectively used for most neutron radiography and tomography stations as a cheaper option than a single crystal bismuth filter. It should also be noted that sapphire filters can be used to improve radiation conditions at experimental stations located on radial channels and to increase the life of the detector system, as well as to improve the quality of neutron imaging data.

\section{Acknowledgments}

This work was supported by a grant from the Plenipotentiary Representative of the Republic of Kazakhstan at JINR, Russia (p.6, No.435 from 31.05.2021).

\section{References}

[1] P. Vontobel et al., Physica B. 385-386 (2006) 475-480. [CrossRef]

[2] I.S. Anderson, R.L. McGreevy, Bilheux H.Z. Neutron imaging and applications: A reference for the imaging community (Springer, New York, 2009) 342 p. [CrossRef]

[3] A.E. Craft et al., Physics Procedia 88 (2017) 73-80. [CrossRef]

[4] A.El. Abd et al., Construction and Building Materials 256 (2020) 119471. [CrossRef]

[5] S.E. Kichanov et al., Physics of Particles and Nuclei Letters 17 (2020) 73-78. [CrossRef]

[6] R.F. Ziesche et al., Nature Communications 11 (2020) 777. [CrossRef]

[7] I.Yu. Zel et al., Acta Geodynamica and Geomateralia 17-3 (2020) 259-267. [CrossRef]

[8] N. Kardjilov, G. Festa, Neutron Methods for Archaeology and Cultural Heritage (Springer, Cham, Switzerland, 2017) 349 p. [CrossRef]

[9] E.H. Lehmann et al., Neutron News 26 (2015) 6-10. [CrossRef]

[10] E.H. Lehmann et al., Physics Procedia 69 (2015) 10-17. [CrossRef]

[11] V.A. Somenkov et al., Journal of Surface Investigation 13 (2019) 870-876. [CrossRef]

[12] Z. Kis et al., Physics Procedia 69 (2015) 40-47. [CrossRef]

[13] K. Nazarov et al., Nuclear Instruments and Methods in Physics Research Section A 982 (2020) 164572. [CrossRef]

[14] B. Muhametuly et al., Journal of Surface Investigation 13 (2019) 877-879. [CrossRef]

[15] J. Soltes et al., Journal of Physics: Conf. Series 749 (2016) 012041. [CrossRef]

[16] D.S. Hussey et al., Nuclear Instruments and Methods in Physics Research Section A 542 (2011) 9-15. [CrossRef]

[17] D.P. Kozlenko et al., Physics Procedia 69 (2015) 87-91. [CrossRef]

[18] D.P. Kozlenko et al., Phys. Part. Nucl. Lett. 13 (2016) 346-351. [CrossRef]

[19] N. Kardjilov et al., Nuclear Instruments and Methods in Physics Research Section A 651 (2011) 47-52. [CrossRef]

[20] F. Ott et al., Physics Procedia 69 (2015) 67-70. [CrossRef] 
[21] L. Crow et al., Nuclear Instruments and Methods in Physics Research Section A 634 (2011) 71-74. [CrossRef]

[22] W. Kockelmann et al., Physics Procedia 43 (2013) 100-110. [CrossRef]

[23] H.F. Nieman et al., Review of Scientific Instruments 51 (1980) 1299-1303. [CrossRef]

[24] J.P. Barton et al., Non-destructive Testing and Evaluation 16 (2001) 95-110. [CrossRef]

[25] O. Aizawa et al., Journal of Nuclear Science and Technology 23 (1986) 562-564. [CrossRef]

[26] B.M. Rustad et al., Review of Scientific Instruments 36 (1965) 48-54. [CrossRef]

[27] R. Born et al., Nuclear Instruments and Methods in Physics Research Section A 262 (1987) 359-365. [CrossRef]

[28] M. Adib et al., Czech J Phys 55 (2005) 563-578. [CrossRef][29] D.F. Mildner et al., Journal of Applied Crystallography 31 (1998) 835-840. [CrossRef]

[30] M. Adib et al.,Radiation Physics and Chemistry 31 (2003) 81-88. [CrossRef]

[31] M. Adib et al., Annals of Nuclear Energy 29 (2002) 1119-1130. [CrossRef]

[32] T.J. Collins, Biotechniques 43 (2007) 25-30. [CrossRef]

[33] C.A. Schneider et al., Nature Methods 9 (2012) 671-675. [CrossRef]

[34] F. Brun et al., Advanced Structural and Chemical Imaging 3 (2017) 4.

[CrossRef]

[35] M.R. Ay et al., Physica Medica 29 (2013) 249-260. [CrossRef]

[36] H. Alsleem et al., Journal of Medical Radiation Sciences 59 (2012) 49-55.

[CrossRef]

[37] D. Kozlenko et al., Crystals 8 (2018) 49-55. [CrossRef]

[38] A.P. Kaestner et al., Physics Procedia 88 (2017) 258-265. [CrossRef] 